\section{A FORTRAN IV program for generating anagrams and for computing several indices in anagram research}

\section{GILBERT J. HARRIS, EDWARD I. GAVURIN and MICHAEL L. GORDON \\ Herbert H. Lehman College, City University of New York Bronx, New York 10468}

This paper describes a computer program which generates all possible anagrams from any five-letter solution word and which also computes a number of frequently employed predictors of problem difficulty for each anagram. The following discussion outlines these indices and their derivations.

For the most part, anagram researchers utilize fiveletter problems considered to be composed of four successive bigrams that correspond to the pairs of letters found in positions $1-2,2-3,3-4$, and $4-5$ of the letter string. The anagram HICAR, for example, is composed of the bigrams HI, IC, CA, and AR; the respective bigrams of its solution word, CHAIR, are $\mathrm{CH}, \mathrm{HA}, \mathrm{AI}$, and IR.

The relative frequency with which any bigram occurs in English is known as its transitional probability (TP), and the TPs of all possible bigrams have been estimated in tables prepared by Mayzner and Tresselt (1965) and Underwood and Schulz (1960). The former table yields a more sensitive measure of bigram frequency since each $\mathrm{TP}$ value is estimated as a function both of the word length (WL) and of the letter position of the bigram (LP). As a result, the same bigram takes on different values when it appears in different-length letter strings or in different positions of a string of given length. For example, the Mayzner-Tresselt TP is 40 for $\mathrm{CH}$ as the first bigram in a five-letter string (e.g., CHAIR), but the TP is 126 for the same bigram when it is in the last two letter positions in a five-letter string and the TP is 7 for the same bigram in the last position of a six-letter string (e.g., CHURCH). The Underwood-Schulz values, on the other hand, make no adjustment for WL or LP, and thus the same TP value is assigned to $\mathrm{CH}$ in each of the three situations above. Both TP computations, however, are used in anagram research and are frequently cited in the literature.

The TP index most often used to assess or control problem difficulty is obtained by adding the bigram frequencies of the four successive letter pairs (summed $\mathrm{TP}$ ) in the anagram or in the solution word. For example, the four bigram frequencies of $\operatorname{HICAR}(5,5$, $10,13)$ yield a summed TP of 33 , while the summed TP of its solution word, CHAIR $(40,33,40,73)$, is 186. Two related indices that have proved useful in predicting problem difficulty (Gavurin, Note 1) are the transitional probability difference (TPD) and the transi- tional probability ratio (TPR). The former is obtained by subtracting the summed TP for the anagram from the summed TP for the solution word. The latter is calculated by dividing the solution word summed TP by the anagram summed TP. Using the bigram TPs presented above for HICAR, the TPD is $153(186-33=$ $153)$ and the TPR is $3.636(186 / 33=3.636)$.

Recently, Mendelsohn and O'Brien (1974) developed a TP predictor of anagram difficulty in the form of a bigram rank measure. This index is obtained by counting the number of bigrams, in the pool of all bigrams formed by the solution word letters, that exceed in TP value the solution word bigrams. The Mayzner-Tresselt values are used in this index. For example, a total of 20 different bigrams can be formed from the letters of the word CHAIR. Table 1 presents these bigrams and their TP values in each of the four letter positions. Using Table 1, the number of bigram TP values that exceed $\mathrm{CH}$ in LP 1-2 is 10. Similarly, for HA in LP 2-3, AI in LP 34, and IR in LP 4-5, the number of higher values are 11,10 , and 4 , respectively. The four ranks when added together yield a value of 35 , which represents the Mendelsohn-O'Brien summed rank index for the solution word CHAIR.

A factor unrelated to TP that also affects anagram difficulty is letter order. Easy letter orders more closely approximate the solution word in that a minimal number of letter moves are necessary to achieve solution. Thus, if the solution word (e.g., CHAIR) letter order is considered to be 12345 , an easy letter order would be 12435 (CHIAR) and a hard letter order would be 52413 (RHICA).

Table 1

Bigram Pool and Matrix of Bigram Values Generated from the Word CHAIR

\begin{tabular}{lcccc}
\hline Bigram & LP 1-2 & LP 2-3 & LP 2-3 & LP 4-5 \\
\hline CH & $40^{*}$ & 0 & 1 & 126 \\
CA & 29 & 3 & 10 & 0 \\
CI & 2 & 1 & 0 & 0 \\
CR & 20 & 1 & 4 & 0 \\
HC & 0 & 0 & 0 & 0 \\
HA & 22 & $33^{*}$ & 0 & 0 \\
HI & 5 & 194 & 0 & 0 \\
HR & 0 & 37 & 0 & 0 \\
AC & 1 & 14 & 51 & 0 \\
AH & 6 & 0 & 0 & 0 \\
AI & 2 & 21 & $40^{*}$ & 0 \\
AR & 8 & 51 & 102 & 13 \\
IC & 1 & 5 & 129 & 18 \\
IH & 0 & 0 & 0 & 0 \\
IA & 0 & 1 & 0 & 0 \\
IR & 1 & 70 & 12 & $73 *$ \\
RC & 0 & 0 & 3 & 0 \\
RH & 0 & 0 & 0 & 0 \\
RA & 23 & 50 & 3 & 2 \\
RI & 50 & 49 & 2 & 0 \\
\hline
\end{tabular}

*Denotes solution word bigrams that are not counted when computing summed rank index. 
Research on the solution of anagrams is a timeconsuming endeavor requiring laborious preliminary preparation in the permutation of solution word letters to form anagrams and in the calculation and determination of a variety of indices considered to be predictors or problem difficulty. A computer program has been written which greatly reduces and simplifies this work.

Input. The input to the program is any number of five-letter solution words and the table TP values from both the Mayzner and Tresselt (1965) and Underwood and Schulz (1960) compilations.

Output. Each of the 120 anagram permutations is generated for each input solution word and the following is provided for each of the permutations: (a) the four individual TP values and the summed TP value using Mayzner and Tresselt (1965), (b) the transitional probability difference using Mayzner and Tresselt (1965), (c) the transtitional probability ratio using Mayzner and Tresselt (1965), (d) the summed TP value using Underwood and Schulz (1960), (e) the letter order. Also provided, but constant across all 120 permutations of a given anagram solution word, is the bigram summed rank index using the Mendelsohn and O'Brien (1974) method.
Computer and Language. The program is written in FORTRAN IV and has been run on an IBM Model 370/168 computer system.

Availability. A copy of the program listing with a sample of output can be obtained free of charge from Gilbert J. Harris, Department of Psychology, Herbert H. Lehman College, City University of New York, Bedford Park Boulevard West, Bronx, New York 10468.

\section{REFERENCE NOTE}

1. Gavurin, E. I. Indices for predicting the solution of fiveletter anagrams. Manuscript in preparation, 1978.

\section{REFERENCES}

Mayzner, M. S., \& Tresselt, M. E. Tables of single-letter and digram frequency counts for various word-length and letter position combinations. Psychonomic Science Monograph Supplements, 1965, 1, 13-31.

Mendelsoin, G. A., \& O'Brien, A. T. The solution of anagrams: $A$ reexamination of the effects of transition letter probabilities, letter moves, and word frequency on anagram difficulty. Memory \& Cognition, 1974, 2, 566-574.

UNDERWOOD, B. J., \& Schulz, R. W. Meaningfulness and verbal learning. Philadelphia: Lippincott, 1960.

(Accepted for publication April 14, 1978.) 